\title{
Outage performance of downlink NOMA-aided small cell network with wireless power transfer
}

\author{
Anh-Tu Le ${ }^{1}$, Dinh-Thuan Do ${ }^{2}$, Munyaradzi Munochiveyi ${ }^{3}$ \\ ${ }^{1,2}$ Faculty of Electronics Technology, Industrial University of Ho Chi Minh City, Vietnam \\ ${ }^{3}$ Department of Computer Science and Information Engineering, College of Information and Electrical Engineering, Asia \\ University, Taiwan
}

\begin{tabular}{l}
\hline Article Info \\
\hline Article history: \\
Received Mar 30, 2021 \\
Revised May 30, 2021 \\
Accepted Jul 28, 2021 \\
\hline
\end{tabular}

\section{Keywords:}

Non-orthogonal multiple access

Outage probability

Power beacon

Small cell network

\begin{abstract}
This article considers the outage performance of the downlink transmission for a small cell network in a heterogeneous network. Due to mobility and distribution of users, it is necessary to study massive connections and high energy efficiency for such kind of systems. To be an enabler of energy harvesting, a power beacon is helpful to support the base station to send signals to distant users, and wireless power transfer (WPT) is exploited to guarantee the data packets transmission from the power beacon to the base station. To provide massive connections, we propose a novel non-orthogonal multiple access (NOMA) technique combined with WPT to enhance outage performance and latency reduction. Furthermore, we derive outage probability (OP) to characterize the system performance. Simulation results are verified to match well between theoretical and analytical methods, and main parameters are determined to understand how they affect the proposed scheme.
\end{abstract}

This is an open access article under the CC BY-SA license.

\section{Corresponding Author:}

Dinh-Thuan Do

Faculty of Electronics Technology

Industrial University of Ho Chi Minh City

12 Nguyen Van Bao, Go Vap Dist., Ho Chi Minh City, Vietnam

Email: dodinhthuan@iuh.edu.vn

\section{INTRODUCTION}

To power the enormous number of internet of things (IoT), energy harvesting (EH) of radio frequency (RF) signals is billed as a promising solution [1]-[3]. In particular, the IoT receivers divide the received signal into two parts (EH and information processing (IP)). Power splitting (PS) or time switching (TS) are two commonly used EH architectures [4], [5]. Studies have shown that PS outperforms TS although it is difficult and inefficient to implement in practice. Recently in [4], [6] and [7], the authors demonstrated how harvested energy contributes to prolonging the lifetime of IoT systems. Reference [6] considered how to secure harvested residual energy after every forwarding and receiving cycle. As such, EH is essential for green and sustainable operation. Also, the authors examined the trade-off between residual harvested energy and the achievable rate. Differently, we go further in this paper to investigate the rate-energy (R-E) region for achievable rate and harvested residual energy in a two-way decode-and-forward (DF) relay system with a PS based energy harvesting relay.

Nowadays, power domain non-orthogonal multiple access (PD-NOMA) has been widely studied by researchers [8]-[14]. The basic principle of PD-NOMA is that the users can transmit power via non-orthogonal transmission while the successive interference cancellation (SIC) receiver is employed at the receiver to miti- 
gate interference [15]-[18]. Further, combining NOMA and cognitive radio (CR) is proposed to offer uninterrupted, reliable, and massive connectivity [16]. Do et al. in [16], the problem of relay selection in CR-NOMA networks when spectrum sharing is operational is considered. As such, the number of accessible secondary users (SUs) sharing the limited and dynamic spectrum resources is boosted due to the opportunistic access of the licensed spectrum resources. Zaidi et al. in [17] studied the use of EH to maintain distinguishable levels of power when the users perform uplink NOMA transmissions. They derived an exact system ergodic average rate to determine the proposed network performance.

The authors Gong and Chen in [19] considered various energy harvesting methods deployed in NOMA systems. They studied several protocols such as conventional TS, PS, and hybrid time-switching/powersplitting (TS/PS) by exploiting a single antenna base station and a single user pair setup. The study in [20] considered WPT via PS and information transmission in a NOMA system consisting of a single antenna base station. A full-duplex information transmitter subject to energy-constraints is studied in [21] to enable energy harvesting from a dedicated energy transmitter while NOMA is employed at the transmitter to communicate with the receivers. Reference [22] examined the performance of a CR-NOMA enabled wireless information and power transfer network (WIPT). Motivated by the above analysis, we deploy a power beacon (PB) to a small cell network in the downlink.

Specifically, to implement IoT relay systems in practice, we analyze TS relaying protocol to facilitate energy harvesting in NOMA systems with the help of a nearby power beacon. This is beneficial for powering IoT devices and sensors such as indicators, alarms, and detectors which are used in underground trains, coal mines, and tunnels. Concerning the large number of IoT nodes in such environments, battery replacement of the sensor nodes or IoT devices is a staggeringly difficult task since connecting all of them through wired connections is difficult. Therefore, it is easy to recharge the IoT sensor nodes by deploying NOMA to transmit data to multiple IoT users. The main benefits of such a proposal is the enhancement of the power consumption, spectral efficiency and capacity demands in hard to reach environments [23]-[32]. Moreover, power beacons can be deployed everywhere to enable wireless power charging of the numerous IoT devices and sensors in these environments. In addition, it is important to provide the performance evaluation of a downlink system using NOMA when the impact of a power beacon is considered in such environments. It is expected that our proposed system model can be implemented in several scenarios as discussed to address existing difficulties.

For the proposed model, we consider first the WPT scenario, and then we present the performance when PB-NOMA is exploited. For such a scenario, we detail comprehensively the outage performances and derive the exact outage probability expressions. Next, we determine which parameter affects the system performance under the constraints of the base station transmit signal-to-noise ratio (SNR) and harvested energy. Our contributions are listed is being as:

- We study PB-NOMA by exploiting the TS scheme to determine the downlink performance under the scheme of linear energy harvesting. NOMA enables the small cell networks to continue their operations if they can accurately capture enough energy in the phase of the energy harvesting process. To facilitate any placement of the base station, the design of the PB benefits nearby base stations to serve their users in the downlink in limited conditions related to power provided by another source.

- We formulate exact outage probability (OP) formulas subject to the constraint of the transmit SNR. We evaluate outage in some cases to show how PB-NOMA guarantees operation in the downlink.

- Simulation results demonstrate that the performance of the proposed scheme in terms of the OP, can be improved if the channel parameters, transmit SNR, and power allocation factors are reasonably selected.

The rest of this paper is organized is being as. Section 2 describes the PB-NOMA small cell networks. In section 3, we consider the scenario of PB-NOMA in terms of outage performance. We provide extensive numerical simulations in section 4 and section 5 concludes the paper.

\section{SIGNAL ANALYSIS AND SYSTEM MODEL}

\subsection{System model}

As can be seen in Figure 1, we consider a small cell network containing an access point (S) which needs the help of a power beacon (PB) to serve two users $\left(U_{i}, i \in\{1,2\}\right)$. In this model, the main nodes ( $\mathrm{S}$, $\mathrm{PB}$, and two user $U_{i}$ ) are equipped with single antennas. Further, all channel are assumed to be identically and independently distributed (i.i.d.) and following Nakagami- $m$ distribution. The signal frame $T$ includes two time slots, shown in Figure 2. The access point $\mathrm{S}$ harvests energy from the beacon in the first time slot $(\alpha T / 2)$

Outage performance of downlink NOMA-aided small cell network with wireless power transfer (Anh-Tu Le) 
while signal processing in the link of $\mathrm{S}-U_{i}$ is conducted in the second time slot $((1-\alpha) T / 2)$. Table 1 contains details of the main system parameters.

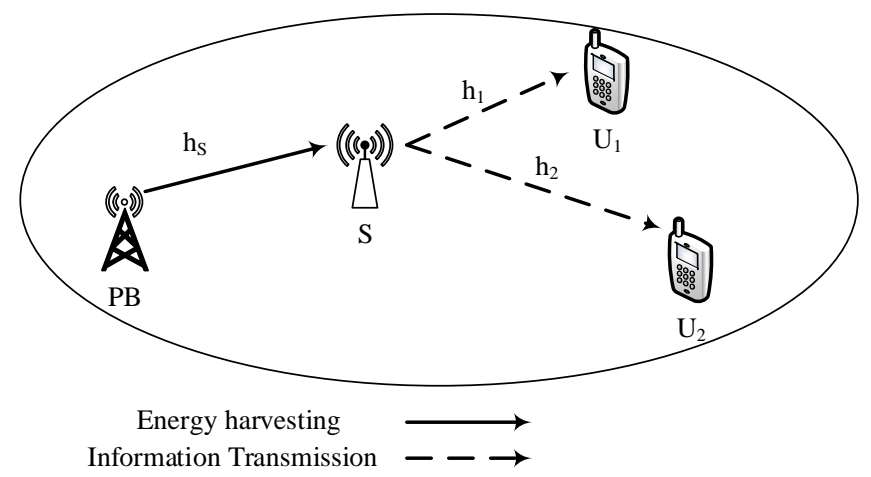

Figure 1. NOMA-aided WPT downlink small cell network

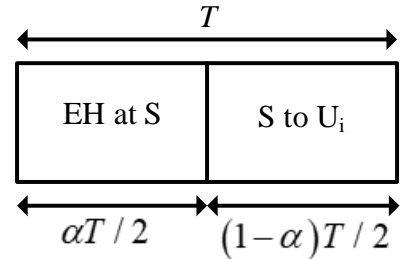

Figure 2. Frame representing energy harvesting and information processing

Table 1. Main parameters

\begin{tabular}{lc}
\hline Symbol & Description \\
\hline$h_{S}$ & The power beacon to base station channel coefficients \\
$h_{1}$ & The base station to $U_{1}$ channel coefficients \\
$h_{2}$ & The base station to $U_{2}$ channel coefficients \\
$P_{B}$ & The power beacon transmit power \\
$P_{S}$ & The base station transmit power \\
$\vartheta$ & The energy conversion efficiency at the power beacon with $0<\vartheta<1$ \\
$x_{i}$ & The information symbol of the user $U_{i}$ with $i \in\{1,2\}$ \\
$\phi_{i}$ & The power allocation factors with $\phi_{1}^{2}>\phi_{2}$ and $\phi_{1}^{2}+\phi_{2}^{1}=1$ \\
$n_{i}$ & The additive white Gaussian noise with $\mathcal{C N}\left(0, \sigma_{i}^{2}\right)$
\end{tabular}

Moreover, the harvested energy at the small cell access point $S$ is formulated by

$$
E_{S}=\frac{\vartheta \alpha P_{B}\left|h_{S}\right|^{2} T}{2}
$$

Then, the average transmit power at $\mathrm{S}$ after the first time slot is obtained as

$$
P_{S}=\frac{E_{S}}{(1-\alpha) T / 2}=\frac{\vartheta \alpha P_{B}\left|h_{S}\right|^{2}}{(1-\alpha)} .
$$

During the second phase, $\mathrm{S}$ transmits the information $\sqrt{P_{S}} \phi_{1} x_{1}+\sqrt{P_{S}} \phi_{2} x_{2}$ to two users $U_{i}$. The $U_{i}$ received signal is computed by

$$
y_{U_{i}}=\sqrt{P_{S}}\left(\phi_{1} x_{1}+\phi_{2} x_{2}\right) h_{i}+n_{i}
$$


Then, the signal-to-interference- plus-noise ratio (SINR) to detect $x_{2}$ at $U_{1}$ is written as

$$
\Gamma_{1,2}=\frac{P_{S} \phi_{1}^{2}\left|h_{1}\right|^{2}}{P_{S} \phi_{2}^{2}\left|h_{1}\right|^{2}+\sigma_{1}^{2}}
$$

Putting (2) into (4), we can rewrite SINR as

$$
\Gamma_{1,2}=\frac{\vartheta \alpha \rho\left|h_{S}\right|^{2}\left|h_{1}\right|^{2} \phi_{1}^{2}}{\vartheta \alpha \rho\left|h_{S}\right|^{2}\left|h_{1}\right|^{2} \phi_{2}^{2}+(1-\alpha)},
$$

where $\rho=\frac{P_{B}}{\sigma_{i}^{2}}$ is the transmit SNR at the access point S.

After applying SIC, the SINR required to detect $x_{1}$ at $U_{1}$ is formulated by

$$
\Gamma_{1}=\frac{\vartheta \alpha \rho\left|h_{S}\right|^{2}\left|h_{1}\right|^{2} \phi_{2}^{2}}{(1-\alpha)} .
$$

Similarly, the SINR to detect $x_{2}$ at $U_{2}$ is formulated as

$$
\begin{aligned}
& \Gamma_{2}=\frac{P_{S} \phi_{1}^{2}\left|h_{2}\right|^{2}}{P_{S} \phi_{2}^{2}\left|h_{2}\right|^{2}+\sigma_{2}^{2}} \\
& =\frac{\vartheta \alpha \rho\left|h_{S}\right|^{2}\left|h_{2}\right|^{2} \phi_{1}^{2}}{\vartheta \alpha \rho\left|h_{S}\right|^{2}\left|h_{2}\right|^{2} \phi_{2}^{2}+(1-\alpha)} .
\end{aligned}
$$

These computations of SINR are the important step to further evaluate OP. It is noted that such outage performance depends on distribution of wireless channels. We then examine such metric along with related impacts in next the section.

\section{OUTAGE PROBABILITY ANALYSIS}

\subsection{Channel distribution}

In this section, $h_{S}$ and $h_{i}$ are followed by the Nakagami-m fading distribution. In particular, the probability density function (PDF) is given as

$$
f_{\left|h_{Q}\right|^{2}}(x)=\frac{1}{\Gamma\left(m_{Q}\right)}\left(\frac{m_{Q}}{\lambda_{Q}}\right)^{m_{Q}} x^{m_{Q}-1} e^{-\frac{m_{Q}}{\lambda_{Q}} x},
$$

where $Q \in\{S, 1,2\}, m_{S}$ and $\lambda_{Q}$ denote the fading severity factor and mean, respectively.

\subsection{Outage probability}

Similar metrics considered in the literature [10]-[16], utilize outage performance as a tool to decide if an emerging technique can be implemented in practice or not. In particular, the OP of $U_{1}$ is defined as [23]

$$
P_{\text {out }, 1}=1-\operatorname{Pr}\left(\Gamma_{1}>\gamma_{1}\right)
$$

where $\gamma_{i}=2^{2 R_{i} /(1-\alpha)}-1$ and $R_{i}$ are the threshold SINR and the target rate, respectively. Substituting (6) into (9), it can be written such that OP for user $U_{1}$ is

$$
\begin{aligned}
P_{\text {out }, 1}= & -\operatorname{Pr}\left(\frac{\vartheta \alpha \rho \phi_{2}^{2}\left|h_{1}\right|^{2}\left|h_{S}\right|^{2}}{(1-\alpha)}>\gamma_{1}\right) \\
& =1-\operatorname{Pr}\left(\left|h_{1}\right|^{2}>\frac{\gamma_{1}(1-\alpha)}{\vartheta \alpha \rho \phi_{2}^{2}\left|h_{S}\right|^{2}}\right) \\
& =1-\int_{0}^{\infty} f_{\left|h_{S}\right|^{2}}(y) \int_{\frac{\gamma_{1}(1-\alpha)}{\vartheta \alpha \rho \phi_{2}^{2} y}}^{\infty} f_{\left|h_{1}\right|^{2}}(x) d x d y
\end{aligned}
$$


With the help of (8), we can express OP as

$$
\begin{aligned}
P_{\text {out }, 1}=1 & -\frac{1}{\Gamma\left(m_{1}\right) \Gamma\left(m_{S}\right)}\left(\frac{m_{1}}{\lambda_{1}}\right)^{m_{1}}\left(\frac{m_{S}}{\lambda_{S}}\right)^{m_{S}} \\
& \times \int_{0}^{\infty} x^{m_{S}-1} e^{-\frac{m_{S}}{\lambda_{S}} x} \int_{\frac{\gamma_{1}(1-\alpha)}{\vartheta \alpha \rho \phi_{2}^{2} y}}^{\infty} x^{m_{1}-1} e^{-\frac{m_{1}}{\lambda_{1}} x} d x d y
\end{aligned}
$$

Based on [33] in (12), we obtain

$$
\begin{aligned}
P_{\text {out }, 1}= & -\sum_{k=0}^{m_{1}-1} \frac{\left(m_{S} / \lambda_{S}\right)^{m_{S}}}{\Gamma\left(m_{S}\right) k !}\left(\frac{m_{1} \gamma_{1}(1-\alpha)}{\lambda_{1} \vartheta \alpha \rho \phi_{2}^{2}}\right)^{k} \\
& \times \int_{0}^{\infty} y^{m_{S}-k-1} e^{-\frac{m_{S}}{\lambda_{S}} y-\frac{m_{1} \gamma_{1}(1-\alpha)}{\lambda_{1} \vartheta \alpha \rho \phi_{2}^{2} y}} d y
\end{aligned}
$$

Moreover, by applying [33] in (13) and (14) the closed-form OP of $U_{1}$ is obtained by

$$
\begin{aligned}
P_{\text {out }, 1}= & -\sum_{k=0}^{m_{1}-1} \frac{2}{\Gamma\left(m_{S}\right) k !}\left(\frac{m_{1} m_{S} \gamma_{1}(1-\alpha)}{\lambda_{1} \lambda_{S} \vartheta \alpha \rho \phi_{2}^{2}}\right)^{\frac{m_{S}+k}{2}} \\
& \times K_{m_{S}-k}\left(2 \sqrt{\frac{m_{1} m_{S} \gamma_{1}(1-\alpha)}{\lambda_{1} \lambda_{S} \vartheta \alpha \rho \phi_{2}^{2}}}\right)
\end{aligned}
$$

Next, the OP of $U_{2}$ is given as [23]

$$
P_{\text {out }, 2}=1-\operatorname{Pr}\left(\Gamma_{1,2}>\gamma_{2}, \Gamma_{2}>\gamma_{2}\right)
$$

Using (5) and (7), OP for user $U_{2}$ as seen in (15) can be expressed as

$$
\begin{aligned}
P_{\text {out }, 2} & =1-\operatorname{Pr}\left(\frac{\vartheta \alpha \rho\left|h_{S}\right|^{2}\left|h_{1}\right|^{2} \phi_{1}^{2}}{\vartheta \alpha \rho\left|h_{S}\right|^{2}\left|h_{1}\right|^{2} \phi_{2}^{2}+(1-\alpha)}>\gamma_{2}, \frac{\vartheta \alpha \rho\left|h_{S}\right|^{2}\left|h_{2}\right|^{2} \phi_{1}^{2}}{\vartheta \alpha \rho\left|h_{S}\right|^{2}\left|h_{2}\right|^{2} \phi_{2}^{2}+(1-\alpha)}>\gamma_{2}\right) \\
& =1-\operatorname{Pr}\left(\left|h_{1}\right|^{2}>\frac{\gamma_{2}(1-\alpha)}{\vartheta \alpha \rho\left(\phi_{1}^{2}-\gamma_{2} \phi_{2}^{2}\right)\left|h_{S}\right|^{2}},\left|h_{2}\right|^{2}>\frac{\gamma_{2}(1-\alpha)}{\vartheta \alpha \rho\left(\phi_{1}^{2}-\gamma_{2} \phi_{2}^{2}\right)\left|h_{S}\right|^{2}}\right) \\
& =1-\int_{0}^{\infty} f_{\left|h_{S}\right|^{2}}(x) \int_{\frac{\gamma_{2}(1-\alpha)}{\vartheta \alpha \rho\left(\phi_{1}^{2}-\gamma_{2} \phi_{2}^{2}\right) x}}^{\infty} f_{\left|h_{1}\right|^{2}}(y) \int_{\frac{\gamma_{2}(1-\alpha)}{\vartheta \alpha \rho\left(\phi_{1}^{2}-\gamma_{2} \phi_{2}^{2}\right) x}}^{\infty} f_{\left|h_{2}\right|^{2}}(z) d z d y d x
\end{aligned}
$$

Thus, with the help of (8), $P_{\text {out }, 2}$ is expressed as

$$
\begin{aligned}
& P_{\text {out }, 2}=1-\frac{\left(m_{S} / \Omega_{S}\right)^{m_{S}}\left(m_{1} / \Omega_{1}\right)^{m_{1}}\left(m_{2} / \Omega_{2}\right)^{m_{2}}}{\Gamma\left(m_{S}\right) \Gamma\left(m_{1}\right) \Gamma\left(m_{2}\right)} \\
& \times \int_{0}^{\infty} x^{m_{S}-1} e^{-\frac{m_{S}}{\Omega_{S}} x} \int_{\frac{\gamma_{2}(1-\alpha)}{\vartheta \alpha \rho\left(\varphi_{1}^{2}-\gamma_{2} \varphi_{2}^{2}\right) x}}^{\infty} y^{m_{1}-1} e^{-\frac{m_{1}}{\Omega_{1}} y} \\
& \times \int_{\frac{\gamma_{2}(1-\alpha)}{\vartheta \alpha \rho\left(\varphi_{1}^{2}-\gamma_{2} \varphi_{2}^{2}\right) x}}^{\infty} z^{m_{2}-1} e^{-\frac{m_{2}}{\Omega_{2}} z} d z d y d x .
\end{aligned}
$$


Following similar steps as before, we can write $P_{\text {out }, 2}$ as (17). Moreover, (17) is express as

$$
\begin{aligned}
P_{\text {out }, 2} & =1-\frac{1}{\Gamma\left(m_{S}\right)}\left(\frac{m_{S}}{\lambda_{S}}\right)^{m_{S}} \sum_{k_{1}=0}^{m_{1}-1} \sum_{k_{2}=0}^{m_{2}-1} \frac{1}{k_{1} ! k_{2} !}\left(\frac{m_{1}}{\lambda_{1}}\right)^{k_{1}}\left(\frac{m_{2}}{\lambda_{2}}\right)^{k_{2}}\left(\frac{\gamma_{2}(1-\alpha)}{\vartheta \alpha \rho\left(\phi_{1}^{2}-\gamma_{2} \phi_{2}^{2}\right)}\right)^{k_{1}+k_{2}} \\
& \times \int_{0}^{\infty} x^{m_{S}-k_{1}-k_{2}-1} e^{-\frac{m_{S}}{\lambda_{S}} x-\frac{\gamma_{2}(1-\alpha)\left(m_{1} \lambda_{2}-\lambda_{1} m_{2}\right)}{\lambda_{1} \lambda_{2} \vartheta \alpha \rho\left(\phi_{1}^{2}-\gamma_{2} \phi_{2}^{2}\right) x}}
\end{aligned}
$$

Finally, the expression of OP in closed-form at user $U_{2}$ is formulated as (18), it is shown as

$$
\begin{aligned}
P_{\text {out }, 2}= & -\sum_{k_{1}=0}^{m_{1}-1} \sum_{k_{2}=0}^{m_{2}-1} \frac{2\left(\Omega_{1} \Omega_{2}\right)^{k_{1}+k_{2}}\left(m_{1} / \Omega_{1}\right)^{k_{1}}\left(m_{2} / \Omega_{2}\right)^{k_{2}}}{k_{1} ! k_{2} ! \Gamma\left(m_{S}\right)\left(m_{1} \Omega_{2}+\Omega_{1} m_{2}\right)^{k_{1}+k_{2}}} \\
& \times\left(\frac{m_{S} \gamma_{2}(1-\alpha)\left(m_{1} \Omega_{2}+\Omega_{1} m_{2}\right)}{\Omega_{S} \Omega_{1} \Omega_{2} \vartheta \alpha \rho\left(\varphi_{1}^{2}-\gamma_{2} \varphi_{2}^{2}\right)}\right)^{\frac{m_{S}+k_{1}+k_{2}}{2}} \\
& \times K_{m_{S}-k_{1}-k_{2}}\left(2 \sqrt{\frac{m_{S} \gamma_{2}(1-\alpha)\left(m_{1} \Omega_{2}+\Omega_{1} m_{2}\right)}{\Omega_{S} \Omega_{1} \Omega_{2} \vartheta \alpha \rho\left(\varphi_{1}^{2}-\gamma_{2} \varphi_{2}^{2}\right)}}\right)
\end{aligned}
$$

\subsection{Throughput}

Relying on OP computed in the previous section, throughput in delay-limited transmission mode is further investigated. At fixed rates $R_{1}, R_{2}$, the throughput can be examined by [30]

$$
\mathcal{T}=\left(1-P_{\text {out }, 1}\right) R_{1}+\left(1-P_{\text {out }, 2}\right) R_{2}
$$

\section{NUMERICAL RESULT AND EVALUATIONS}

In this section, we present the numerical analysis of our PB-NOMA system along with corroboration of analytical results. The parameters can be seen in Table 2. Figure 3 depicts OP for two users $U_{1}, U_{2}$ versus transmit SNR at the access point. Such outage performance can be improved when the system achieves high SNR. The reason is that high transmit power at the access point leads to high SNR, then outage performance is enhanced significantly, especially in high SNR region. At different target rates, the system shows two different curves of outage probability. It can be seen that at lower target rates, the better the OP can be achieved.

Figure 4 examines how the factor $m$ influences to OP for two users $U_{1}, U_{2}$. The similar trends of OP can be seen in this figure when compared with Figure 3. It is concluded that $m$ affects performance, $m=3$ exhibits the best case. The performance gap of the two users exists due to different power allocation factors. It can be seen that the system continues its operation as normal if OP is kept low.

Figure 5 provides outage performance at the range of percentage time for energy harvesting. It is obvious that the more time is allocated to energy harvesting leads to higher transmit power at the access point, then such outage performance can be improved. We have similar trends of OP as in previous figures. Similar performance can be seen in Figure 6 as varying $\vartheta$. The main reason is that $\vartheta$ contributes to how much energy can be harvested at the access point and the OP will be changed as well.

Table 2. Numerical result parameters

\begin{tabular}{ll}
\hline Target rates & $R_{1}=R_{2}=0.4$ \\
\hline Power allocation coefficients & $\varphi_{1}^{2}=0.8, \varphi_{1}^{2}=0.2$ \\
The fading severity factor mean & $m_{S}=m_{1}=m_{2}=m=2$ \\
& and $\lambda_{S}=\lambda_{2}=1, \lambda_{1}=2$ \\
& $\vartheta=0.9$ \\
The energy conversion efficiency & $10^{6}$ \\
Monte Carlo simulation &
\end{tabular}

Outage performance of downlink NOMA-aided small cell network with wireless power transfer (Anh-Tu Le) 


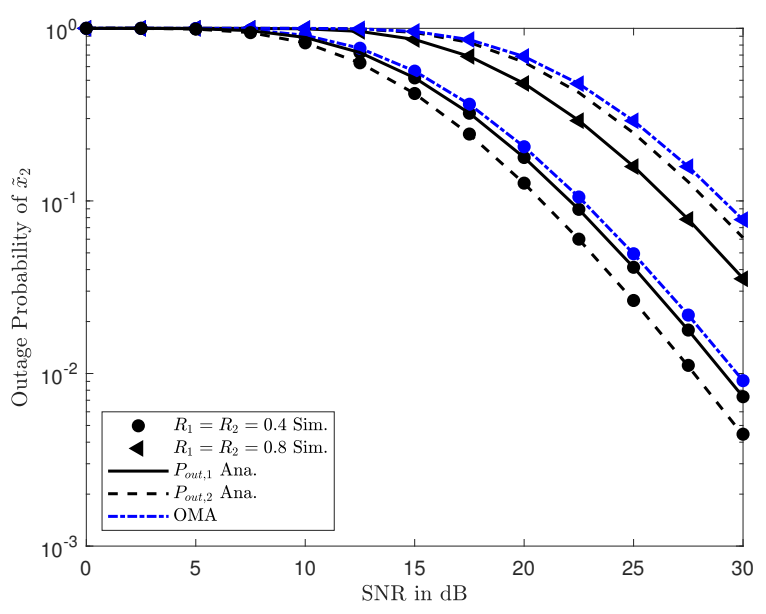

Figure 3. The outage probability versus SNR varying $R_{1}=R_{2}$ with $\alpha=0.1$

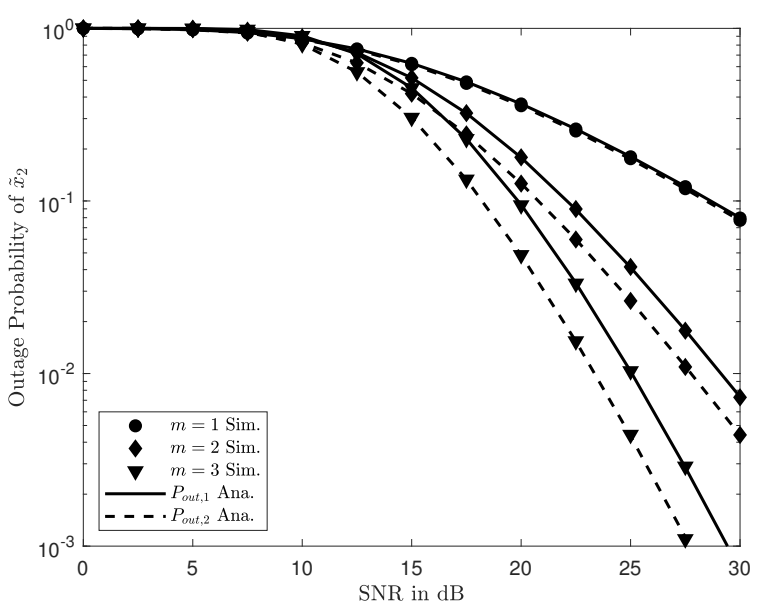

Figure 4. The outage probability versus SNR varying $m$ with $\alpha=0.1$

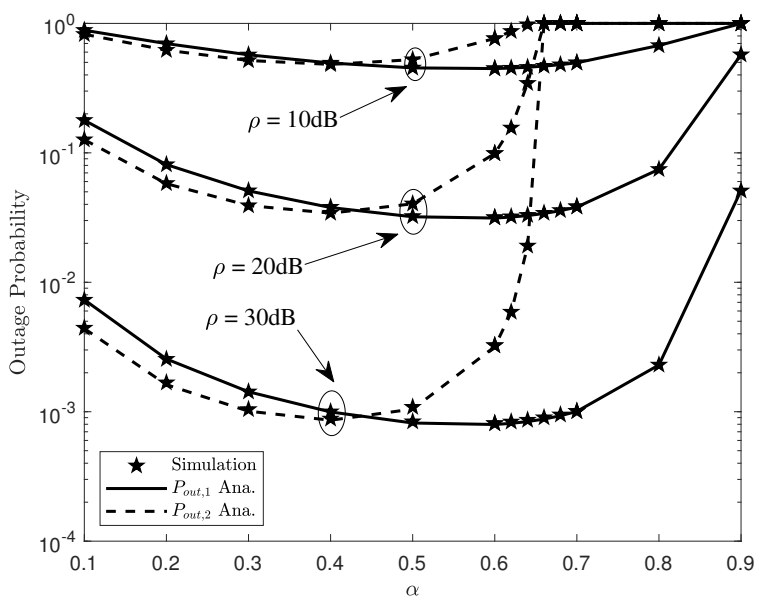

Figure 5. The outage probability versus $\alpha$ varying $\rho$ with $\alpha=0.1$ 


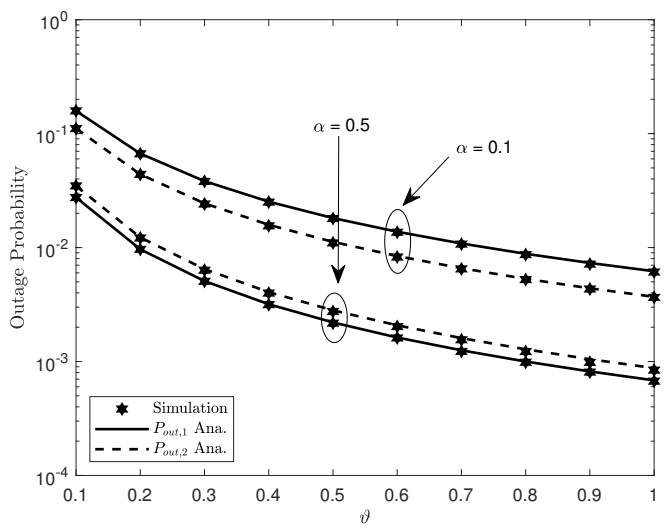

Figure 6. The outage probability versus $\vartheta$ varying $\alpha$ with $\rho=30 \mathrm{~dB}$

Figure 7 indicates that high throughput occurs in the high SNR region. It can be explained by the fact that OP influences throughput. This result is definitely consistent with outage performance reported in previous figures. Similarly to Figure 7 and Figure 8 illustrates how energy harvesting impacts throughput in the whole SNR range. It can be explained by the fact that OP depends on how much energy is harvested and throughput is affected as well. This result provides guidelines to implement energy harvesting in PB-NOMA.

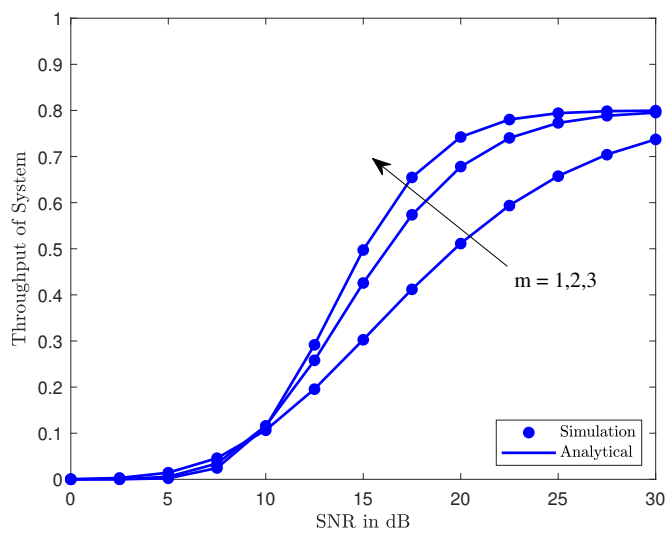

Figure 7. System throughput versus SNR varying $m$ with $\alpha=0.1$

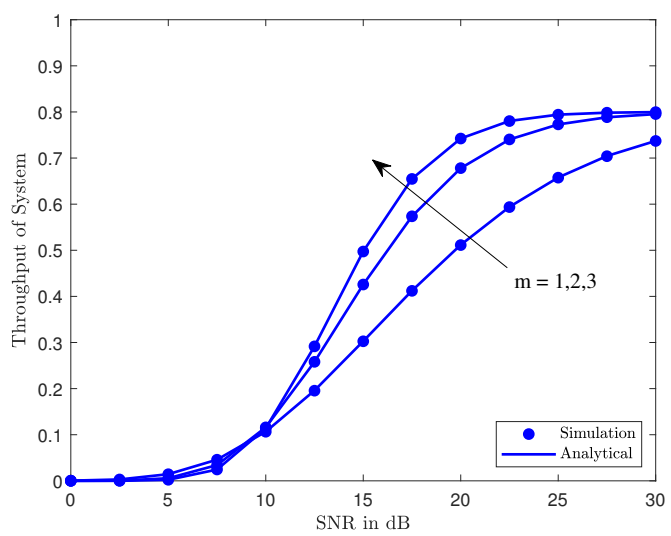

Figure 8. System throughput versus $\vartheta$ varying $\alpha$ with $\rho=20 \mathrm{~dB}$ 


\section{CONCLUSION}

Taking into account the linear characteristics of the actual circuit providing energy harvesting, this paper has presented a mathematical framework to analyze system performance. Taking into account the outage characteristics of the system. Based on the model, since the Nakagami- $m$ channel is adopted, this paper derived the exact expression of the outage probability downlink of PB-NOMA system based on the TS protocol and calculated the throughput of the system. To find parameters affecting to system, this paper provided simulations that verified the outage probability as derived in the theory. The simulation results verified the exactness of the theoretical derivation and showed the influence of the different system parameters on the outage and throughput performance. When the transmission rate are small and the harvested power is high at the access point, the PBNOMA system had better outage throughput. The simulations also showed that there is a gap between two users which is caused by allocated power levels to each user. This study benefits the design of IoT with the ability harvest energy. We can extend the two-user PB-NOMA approach to multiple users in future work.

\section{REFERENCES}

[1] X. Lu, P. Wang, D. Niyato, D. I. Kim, and Z. Han, "Wireless Networks With RF Energy Harvesting: A Contemporary Survey," in IEEE Communications Surveys Tutorials, vol. 17, no. 2, pp. 757-789, Secondquarter 2015, doi: 10.1109/COMST.2014.2368999.

[2] A. A. Nasir, H. D. Tuan, D. T. Ngo, S. Durrani, and D. I. Kim, "Path-Following Algorithms for Beamforming and Signal Splitting in RF Energy Harvesting Networks," in IEEE Communications Letters, vol. 20, no. 8, pp. 1687-1690, Aug. 2016, doi: 10.1109/LCOMM.2016.2578921.

[3] V. Nguyen, T. Q. Duong, H. D. Tuan, O. Shin, and H. V. Poor, "Spectral and Energy Efficiencies in Full-Duplex Wireless Information and Power Transfer," in IEEE Transactions on Communications, vol. 65, no. 5, pp. 2220-2233, May 2017, doi: 10.1109/TCOMM.2017.2665488.

[4] H. H. M. Tam, H. D. Tuan, A. A. Nasir, T. Q. Duong, and H. V. Poor, "MIMO Energy Harvesting in Full-Duplex Multi-User Networks," in IEEE Transactions on Wireless Communications, vol. 16, no. 5, pp. 3282-3297, May 2017, doi: 10.1109/TWC.2017.2679055.

[5] A. A. Nasir, H. D. Tuan, T. Q. Duong, and H. V. Poor, "Secrecy Rate Beamforming for Multicell Networks With Information and Energy Harvesting," in IEEE Transactions on Signal Processing, vol. 65, no. 3, pp. 677-689, February 2017, doi: 10.1109/TSP.2016.2621719.

[6] C. In, H. Kim, and W. Choi, "Achievable Rate-Energy Region in Two-Way Decode-and-Forward Energy Harvesting Relay Systems," in IEEE Transactions on Communications, vol. 67, no. 6, pp. 3923-3935, June 2019, doi: 10.1109/TCOMM.2019.2901783.

[7] A. A. Nasir, H. D. Tuan, T. Q. Duong, and H. V. Poor, "Secure and Energy-Efficient Beamforming for Simultaneous Information and Energy Transfer," in IEEE Transactions on Wireless Communications, vol. 16, no. 11, pp. 75237537, Nov. 2017, doi: 10.1109/TWC.2017.2749568.

[8] D.-T. Do, A.-T. Le, and B.-M. Lee, "On Performance Analysis of Underlay Cognitive Radio-Aware Hybrid OMA/NOMA Networks with Imperfect CSI," Electronics, vol. 8 no. 7, p. 819, 2019, doi: 10.3390/electronics8070819.

[9] D. Do, A. Le, and B. M. Lee, "NOMA in Cooperative Underlay Cognitive Radio Networks Under Imperfect SIC," in IEEE Access, vol. 8, pp. 86180-86195, 2020, doi: 10.1109/ACCESS.2020.2992660.

[10] O. Abbasi, A. Ebrahimi, and N. Mokari, "NOMA Inspired Cooperative Relaying System Using an AF Relay," in IEEE Wireless Communications Letters, vol. 8, no. 1, pp. 261-264, Feb. 2019, doi: 10.1109/LWC.2018.2869592.

[11] E. Khorov, A. Kureev, and I. Levitsky, "NOMA Testbed on Wi-Fi," in 2018 IEEE 29th Annual International Symposium on Personal, Indoor and Mobile Radio Communications (PIMRC), 2018, pp. 1153-1154, doi: 10.1109/PIMRC.2018.8580931.

[12] J. Zeng, et al., "Investigation on Evolving Single-Carrier NOMA Into Multi-Carrier NOMA in 5G," in IEEE Access, vol. 6, pp. 48268-48288, 2018, doi: 10.1109/ACCESS.2018.2868093.

[13] Dinh-Thuan Do, Minh-Sang V. Nguyen, Thi-Anh Hoang, and B. M. Lee, "Exploiting joint base station equipped multiple antenna and full-duplex D2D users in power domain division based multiple access networks," Sensors, vol. 19 , no. 11 , p. 2475 , doi:10.3390/s19112475.

[14] D.-T. Do, A.-T. Le, C.-B. Le, and B. M. Lee "On Exact Outage and Throughput Performance of Cognitive Radio based Non-Orthogonal Multiple Access Networks With and Without D2D Link," Sensors vol. 19, no. 15, p. 3314, 2019, doi: 10.3390/s19153314.

[15] F. Kara and H. Kaya, "On the Error Performance of Cooperative-NOMA With Statistical CSIT," in IEEE Communications Letters, vol. 23, no. 1, pp. 128-131, Jan. 2019, doi: 10.1109/LCOMM.2018.2878729.

[16] D. -T. Do, M. -S. V. Nguyen, F. Jameel, R. Jäntti, and I. S. Ansari, "Performance Evaluation of Relay-Aided CRNOMA for Beyond 5G Communications," in IEEE Access, vol. 8, pp. 134838-134855, 2020, doi: 10.1109/AC- 
CESS.2020.3010842.

[17] S. K. Zaidi, S. F. Hasan, and X. Gui, "Evaluating the Ergodic Rate in SWIPT-Aided Hybrid NOMA," in IEEE Communications Letters, vol. 22, no. 9, pp. 1870-1873, Sept. 2018, doi: 10.1109/LCOMM.2018.2849071.

[18] J. W. Kim, S. Y. Shin, and V. C. M. Leung, "Performance Enhancement of Downlink NOMA by Combination With GSSK," in IEEE Wireless Communications Letters, vol. 7, no. 5, pp. 860-863, Oct. 2018, doi: 10.1109/LWC.2018.2833469.

[19] J. Gong and X. Chen, "Achievable Rate Region of Non-Orthogonal Multiple Access Systems With Wireless Powered Decoder," in IEEE Journal on Selected Areas in Communications, vol. 35, no. 12, pp. 2846-2859, Dec. 2017, doi: 10.1109/JSAC.2017.2726242.

[20] G. He, L. Li, X. Li, W. Chen, L. Yang, and Z. Han, "Secrecy sum rate maximization in NOMA systems with wireless information and power transfer," 2017 9th International Conference on Wireless Communications and Signal Processing (WCSP), 2017, pp. 1-6, doi: 10.1109/WCSP.2017.8171177.

[21] P. Deng, B. Wang, W. Wu, and T. Guo, "Transmitter Design in MISO-NOMA System With Wireless-Power Supply," in IEEE Communications Letters, vol. 22, no. 4, pp. 844-847, April 2018, doi: 10.1109/LCOMM.2018.2799949.

[22] F. Zhou, Z. Chu, H. Sun, R. Q. Hu, and L. Hanzo, "Artificial Noise Aided Secure Cognitive Beamforming for Cooperative MISO-NOMA Using SWIPT," in IEEE Journal on Selected Areas in Communications, vol. 36, no. 4, pp. 918-931, April 2018, doi: 10.1109/JSAC.2018.2824622.

[23] S. K. Zaidi, S. F. Hasan, and X. Gui, "SWIPT-aided uplink in hybrid non-orthogonal multiple access," 2018 IEEE Wireless Communications and Networking Conference (WCNC), 2018, pp. 1-6, doi: 10.1109/WCNC.2018.8376963.

[24] M. Hedayati and I. Kim, "On the Performance of NOMA in the Two-User SWIPT System," in IEEE Transactions on Vehicular Technology, vol. 67, no. 11, pp. 11258-11263, Nov. 2018, doi: 10.1109/TVT.2018.2866612.

[25] A. Rauniyar, P. E. Engelstad, and O. N. Østerbø, "Performance Analysis of RF Energy Harvesting and Information Transmission Based on NOMA With Interfering Signal for IoT Relay Systems," in IEEE Sensors Journal, vol. 19, no. 17, pp. 7668-7682, Sept. 2019, doi: 10.1109/JSEN.2019.2914796.

[26] H. Zhang, M. Feng, K. Long, G. K. Karagiannidis, and V. C. M. Leung, "Energy-Efficient Resource Allocation in NOMA Heterogeneous Networks with Energy Harvesting," 2018 IEEE Global Communications Conference (GLOBECOM), 2018, pp. 206-212, doi: 10.1109/GLOCOM.2018.8647140.

[27] W. Zhao, R. She, and H. Bao, "Security Energy Efficiency Maximization for Two-Way Relay Assisted Cognitive Radio NOMA Network With Self-Interference Harvesting," in IEEE Access, vol. 7, pp. 74401-74411, 2019, doi: 10.1109/ACCESS.2019.2920710.

[28] H. Bao, C. Zhang, L. Wu, and M. Li, "Design of physical layer secure transmission scheme based on SWIPT NOMA systems," 2017 IEEE 17th International Conference on Communication Technology (ICCT), 2017, pp. 6-9, doi: 10.1109/ICCT.2017.8359473.

[29] Z. Na, et al., "Subcarrier allocation based simultaneous wireless information and power transfer algorithm in 5G cooperative OFDM communication systems," Physical Communication, vol 29, pp. 164-170, August 2018, doi: 10.1016/j.phycom.2018.05.008.

[30] Z. Song, X. Wang, Y. Liu, and Z. Zhang, "Joint Spectrum Resource Allocation in NOMA-based Cognitive Radio Network With SWIPT," in IEEE Access, vol. 7, pp. 89594-89603, 2019, doi: 10.1109/ACCESS.2019.2926429.

[31] P. D. Diamantoulakis, K. N. Pappi, Z. Ding, and G. K. Karagiannidis, "Wireless-Powered Communications With Non-Orthogonal Multiple Access," in IEEE Transactions on Wireless Communications, vol. 15, no. 12, pp. 84228436, Dec. 2016, doi: 10.1109/TWC.2016.2614937.

[32] H. Dang, M. V. Nguyen, D. Do, H. Pham, B. Selim, and G. Kaddoum, "Joint Relay Selection, Full-Duplex and Device-to-Device Transmission in Wireless Powered NOMA Networks," in IEEE Access, vol. 8, pp. 82442-82460, 2020, doi: 10.1109/ACCESS.2020.2991847.

[33] I. S. Gradshteyn and I. M. Ryzhik, "Table of Integrals, Series, and Products," 6/e. San Diego, CA: Academic Press, 2000. 